\title{
Osteoarthritis: Pathophysiology, Treatment, and What Muslims Need to Know Prior to Joint Replacement Surgery
}

\author{
Asif M. Ilyas, MD \\ Assistant Professor of Orthopaedic Surgery \\ Temple University Hospital \\ Philadelphia, Pennsylvania
}

\begin{abstract}
Osteoarthritis is the most common type of arthritis. The joints most commonly involved include the knees, hips, fingers and thumbs, and the spine. Risk factors include age ( $>50$ years), obesity, joint hypermobility/instability, prolonged occupational/sports stress, and previous joint injury. The pathogenesis of osteoarthritis is multifactorial, but the ultimate endpoint is the alteration of the articular cartilage. The diagnosis of osteoarthritis can usually be made by a detailed history and physical examination and reliably confirmed by plain radiographs. The primary goals of treatment for osteoarthritis are improved function and quality of life. Treatment modalities include patient education, physical therapy, pharmacotherapy, intra-articular injections, and surgery. Joint replacement surgery has been steadily growing in frequency and success. Hips and knees are the most commonly replaced joints. After successful joint replacement of a Muslim's hips or knees, the resulting range of motion under normal circumstances may not allow sufficient motion to perform salat in the standard format.
\end{abstract}

Key words: Arthritis, joint replacement, joint arthroplasty, prayer.

Pathophysiology and Treatment Introduction

$\mathrm{O}$ steoarthritis is the most common type of arthritis. It is also known as "degenerative joint disease." It is estimated that osteoarthritis affects 40 million Americans of all ages and that 70 to $90 \%$ of Americans 75 years old or older have at least one involved joint. ${ }^{1}$ Estimates of the prevalence of osteoarthritis based on radiographic evidence

Correspondence should be directed to

\section{Asif M. Ilyas, $M D$}

3401 North Broad Street

Philadelphia, PA 19140

Telephone: 215-707-8332

Email:amilyas@temple.edu range from 30 to $90 \% .{ }^{1}$

Men and women are equally affected, but symptoms occur earlier and appear to be more severe in women. The joints most commonly involved include the knees, hips, interphalangeal joints of the fingers, the carpometacarpal joint of the thumb, and the spine (Figure 1).

\section{Etiology}

The term "arthritis" encompasses a heterogeneous group of more than 100 diseases involving the synovial joints and the periarticular structures. ${ }^{2}$ The exact pathogenesis underlying most of these disorders is uncertain, but pathology within the synovium, articular cartilage, and their subcomponents are believed to be the primary cause of the most common types of arthritis. 


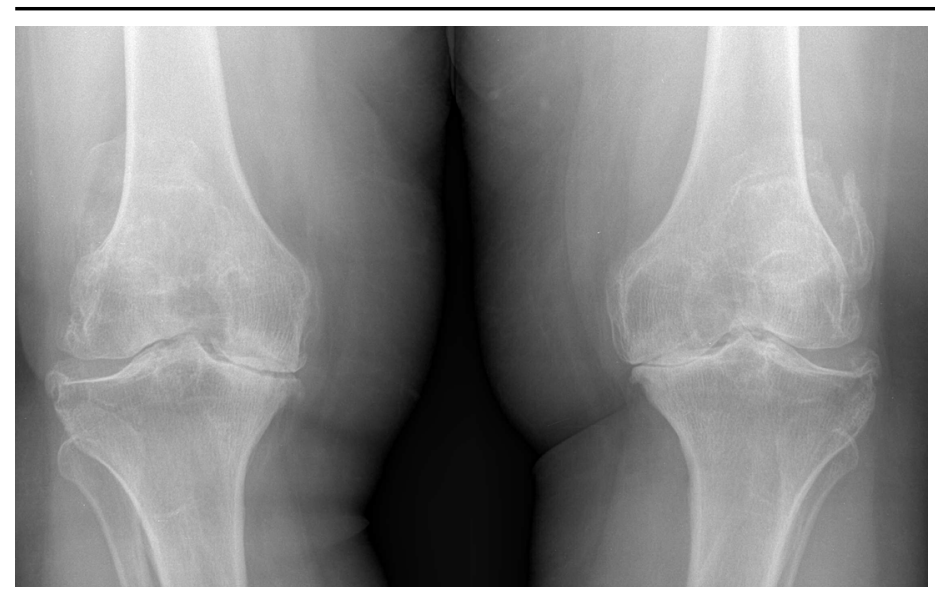

Figure 1. Bilateral knee osteoarthritis on radiographs. Note the joint space narrowing, osteophyte formation and alteration in subchondral bone density.

Osteoarthritis is the most common form of arthritis and similarly the exact etiology of this "primary" arthritis is unknown but is considered to be multifactorial. Risk factors include age ( $>50$ years), obesity, joint hypermobility/instability, prolonged occupational/sports stress, and previous joint injury (Table 1). In contrast, any specific insult that changes the environment within a joint has the potential to cause a "secondary" arthritis (Table 2).

The prevalence of osteoarthritis is greatest in older populations. The increased incidence of osteoarthritis with age is related to progressive ligamentous laxity, decreased function of supporting periarticular soft tissue and proprioceptic function, a reduction in the ability of articular cartilage to remodel, and an overall decrease in the chondrocyte number within the cartilage.

In addition, there is an increased incidence of osteoarthritis with certain occupations. ${ }^{2}$ Farmers and miners have an increased incidence of hip osteoarthritis. Occupations that require regular kneeling or squatting are associated with an increased incidence of knee osteoarthritis. Professional athletes, although not recreational athletes, have been shown to have a higher incidence of knee osteoarthritis as well.

\section{Pathogenesis}

The term osteo-"arthritis" is a misnomer because inflammation is not the primary pathologic process but rather is a combination of factors that result in the degenerative changes of osteoarthritis. The inflammation that does result in osteoarthritis is mild. This is in contrast to rheumatoid arthritis, the
Table 1. Risk factors for osteoarthritis.

Age $>50$ years

Obesity

Joint hypermobility/laxity

Prolonged occupational/sports stress

Previous joint derangement

Table 2. Secondary causes of arthritis.

Crystalline arthropathy

gout

pseudogout

Joint infection

Peripheral neuropathy

diabetes

tabes dorsalis

Metabolic derangement

Wilson's disease

hemochromatosis

Osteonecrosis of the joint

Prolonged joint immobilization

Previous joint injury

intra-articular fracture

physeal injury in childhood

second most common form of arthritis, where autoimmune joint inflammation and destruction is the primary mechanism of joint injury.

The pathogenesis of osteoarthritis is multifactorial, but the ultimate endpoint is the alteration of the articular cartilage. Articular cartilage is composed of water, type II collagen, and proteoglycans. In healthy cartilage, continual internal remodeling occurs as the chondrocytes replace macromolecules lost through degradation associated with normal joint activity. This process becomes impaired in osteoarthritis, leading to increased degenerative changes and an abnormal repair response. The result is altered articular cartilage with increased water content, decreased proteoglycan content, and altered collagen matrix from the more resilient type II to the less durable type I fibrocartilage. 
Table 3. Joint synovial fluid analysis.

\begin{tabular}{lllllll}
\hline $\begin{array}{l}\text { Arthritis } \\
\text { type }\end{array}$ & $\begin{array}{l}\text { White blood } \\
\text { cell count } \\
\left(\text { per } \mathrm{mm}^{3}\right)\end{array}$ & $\begin{array}{l}\text { Polymorphonuclear } \\
\text { leukocytes }(\%)\end{array}$ & Glucose & Protein & Crystals & Cultures \\
\hline $\begin{array}{l}\text { Osteoarthritis } \\
<200\end{array}$ & $<25$ & normal & normal & negative & negative \\
$\begin{array}{l}\text { Inflammatory } \\
\text { arthritis }\end{array}$ & $>2,000$ & 50 & normal & increased & positive & negative \\
$\begin{array}{l}\text { Septic } \\
\text { arthritis }\end{array}$ & $>75,000$ & $>75$ & decreased & increased & negative & positive \\
\hline
\end{tabular}

Table 4. Pharmacotherapy for osteoarthritis.

\begin{tabular}{ll}
\hline Class & Medications \\
\hline Analgesics & acetaminophen (Tylenol) \\
NSAIDs & salicylic acids: aspirin \\
& propionic acids: \\
& ibuprofen (Motrin), naproxen (Naprosyn), Ketoprofen \\
& acetic acids: indomethacin (Indocin) \\
& enolic acids: meloxicam (Mobic), proxicam (Feldene) \\
& napthylalkalanones: nabumetone (Relafen) \\
& pyranocarboxylic acids: Etodolac (Lodine) \\
& pyrroles: ketorolac (Toradol) \\
cyclooxygenase-2 inhibitors & valdecoxib (Bextra) - withdrawn in 2005 \\
rofecoxib (Vioxx) - withdrawn in 2004 & Kenalog \\
\hline
\end{tabular}

The strong association between age and osteoarthritis may further be explained by age-related changes in the matrix composition and a decrease in chondrocyte function and responsiveness to stimuli. These changes interfere with continued internal remodeling, maintenance of the cartilage tissue, and loss of cartilage, leading to an increased risk for cartilage degradation and injury. Furthermore, the abnormal repair response leads to the formation of osteophytes and subchondral cysts as the disease 
progresses. These changes present clinically and are evident on radiographs.

Obesity is an important and modifiable risk factor for bilateral knee osteoarthritis. The association of obesity and knee osteoarthritis is stronger in women than in men. It is postulated that obesity increases the incidence of knee osteoarthritis by increasing the forces across the knee, increasing bone density, and through the release of detrimental growth factors or hormones stored within excess adipose tissue. ${ }^{3}$

\section{Diagnosis}

The diagnosis of osteoarthritis can usually be made by a detailed history and physical examination and reliably confirmed by plain radiographs. The usual presenting symptom is pain involving one or only a few joints. Morning joint stiffness that usually improves within 30-60 minutes of activity is common. Similarly, joint pain and stiffness are common after mild-to-moderate activity. As the disease progresses, prolonged joint stiffness, progressive joint pain, and persistent joint enlargement become evident. Crepitus, or a grating sensation in the joint, is a common manifestation. Heberden's nodes are commonly seen in primary osteoarthritis due to enlargement of the distal interphalangeal joints of the hand. Similar enlargements of the proximal interphalangeal joints of the fingers are called Bouchard's nodes.

Additional diagnostic evaluation is indicated when the diagnosis remains uncertain, response to therapy is not as expected, or the clinical picture varies. Table 2 lists common secondary causes of arthritis that can be differentiated with the use of serum and joint synovial fluid analysis. Serum blood work should include erythrocyte sedimentation rate, c-reactive protein, and rheumatoid factor. In osteoarthritis, the synovial fluid white blood cell count is usually less than 500 cells per $\mathrm{mm}^{3}$ and is composed predominantly of mononuclear cells, which are unlike secondary arthritides that have inflammatory aspirates and synovial fluid white blood cell counts that are typically greater than 2000 cells per $\mathrm{mm}^{3}$. The predominant cell type is usually the neutrophil (Table 3).

Radiographs can readily confirm the diagnosis of osteoarthritis and grade the severity of disease. Radiographic findings consistent with osteoarthritis include the presence of joint space narrowing, osteophyte formation, subchondral bone cyst formation, and increasing density of the subchondral bone (Figure 1). Subchondral bone is defined as the bone sitting closest to the joint surface that is just below the radiolucent articular cartilage. Late findings include bony erosion, joint subluxation, and deformity. Early on, radiographs may be normal for articular damage and take time to evolve. On the same note, many patients with radiographic changes consistent with osteoarthritis are asymptomatic or do not exhibit any disability, suggesting that the presence of radiographic changes alone in the absence of symptoms should not lead to the diagnosis of osteoarthritis.

\section{Treatment}

The primary goals of treatment for osteoarthritis are improved function and quality of life. Treatment should be tailored to the needs of the individual patient and provided in a graded manner relative to the extent of disease and disability. Modalities include the following:

1. Patient education

2. Rehabilitation and activity modification

3. Pharmacotherapy

4. Intra-articular injections/viscosupplementation

5. Surgery

Patients should be thoroughly educated about the natural course of osteoarthritis. The patients' role in the management of the disease is critical. Many studies have shown that effective patient education using arthritis self-management programs results in improved pain and functional scores. ${ }^{2}$ Physical therapy and regular exercise also improve pain scores, function, and avoid overall musculoskeletal deconditioning. Reduced-load and lowimpact exercises, such as cycling or swimming, are most beneficial. In addition, resistance exercises, such as monitored weight-training, do not exacerbate the symptoms of osteoarthritis. ${ }^{4}$

Orthotics can also be beneficial in off-loading arthritis in certain circumstances. Using knee osteoarthritis as an example, osteoarthritis is most commonly involved in the medial compartment; a lateral heel wedge in a shoe can help diminish the load seen across the medial compartment of the 


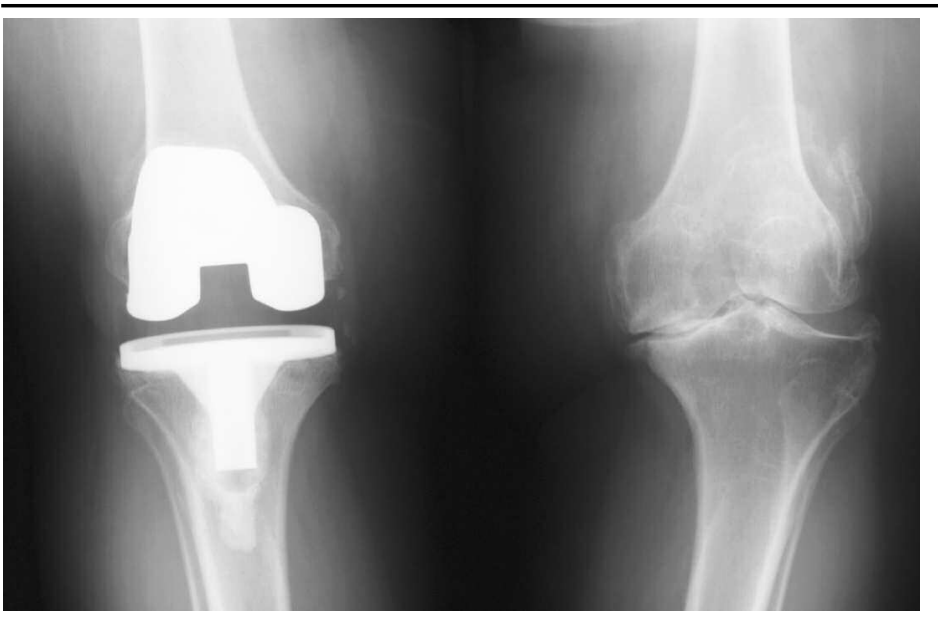

Figure 2. Knee radiograph after total knee replacement. There are changes to the joint range of motion that can result in significant limitations to a patient's ability to perform salat.

knee.

Certain therapies touted in the media or by the allied health fields may provide some symptomatic relief but have not been validated in controlled clinical trials. These include physical therapy modalities such as ultrasound and transcutaneous electrical nerve stimulation. In addition, there is conflicting data on the efficacy of oral supplements such as oral glucosamine to reduce symptoms of joint osteoarthritis. Similarly, the Federal Drug Administration (FDA) does not standardize or regulate their formulations.

Pain is the primary symptom of osteoarthritis, and multiple medications are available to relieve pain and improve function (Table 4). The choice of a pain control medication must be individualized by prescribing medications with the best side effect profile first and adding other pain control medications as indicated. Initial treatment is with acetaminophen (up to $4 \mathrm{~g} /$ day). If this treatment is unsuccessful, or if there are features of local inflammation such as joint warmth or effusion, use of a nonsteroidal anti-inflammatory drug (NSAID) is appropriate. Although osteoarthritis is not considered an inflammatory arthritis the inhibition of prostaglandin E2 does provide relief in osteoarthritic pain. In addition, NSAIDs can provide a direct analgesic effect on the central nervous system.

Although more effective than acetaminophen, NSAID use is tempered by the possibility of gastrointestinal (GI) side effects such as abdominal pain and dyspepsia, and in some cases peptic ulcer disease or its complications of hemorrhage or perforation. Risk factors for GI complications with the use of NSAIDs include age $>60$ years, prior history of a GI event, history of rheumatoid arthritis, concurrent corticosteroid use, and concurrent anticoagulant use. The relative risk of incurring serious GI side effects from chronic NSAID use can be up to 2.25 times above normal. ${ }^{5}$ To avoid GI complications, NSAIDs can be given with proton-pump inhibitor medication or as the newer selective cyclooxygenase-2 inhibitor form.

The selective cyclooxygenase-2 (COX-2) inhibitors provide anti-inflammatory effect but avoid the GI side affects by not interfering with the GI-protective cyclooxygenase- 1 enzymes. The result is a $50 \%$ decreased incidence of GI side effects. ${ }^{6}$ In October 2004, Merck withdrew rofecoxib (Vioxx) and later in April 2005, Pfizer voluntarily withdrew valdecoxib (Bextra) from the market due to their unfavorable cardiovascular profile. Specifically, a randomized control trial with rofecoxib looking at the use of $25 \mathrm{mg}$ in patients with GI polyps found a significantly higher myocardial infarction rate after 18 months: $1.48 \%$ in the rofecoxib group versus $0.75 \%$ in the placebo group. ${ }^{7}$ Therefore, although the effects of coxibs are reversible and dose-dependent, it would seem most reasonable to prescribe them to patients with higher GI and lower cardiovascular risks.

Intra-articular injections remain a common treatment when oral pharmacologic agents are not beneficial or contraindicated. Steroid injections can be administered effectively through multiple techniques and formulations. As a general rule, intraarticular steroid injections should be limited to no more than three to four times per year due to the theoretical risk of further joint deterioration. ${ }^{8}$ Results of a multicenter, randomized study demonstrated that intra-articular knee injections significantly reduced pain for up to four weeks. No functional improvement was noted compared to a placebo. Results from this same study revealed that joint lavage significantly relieved pain for up to 24 weeks. ${ }^{9}$ The effects of steroid injection and joint lavage on pain relief were additive, but neither procedure significantly improved functional status. Sodium hyaluronate (Synvisc, Hyalgan) is indicated for the treatment of patients with only osteoarthritis of the knee. Although side effects are uncommon, the current data is conflicting, and the overall impact is 
small. Synvisc was compared head-to-head with Celestone in a prospective randomized study, and both were found to have no advantage over the other at the 6-month follow-up. ${ }^{10}$

When other modalities fail, surgical intervention for osteoarthritis is available with the goal of eliminating pain and improving function. A spectrum of surgical options exists, ranging from soft tissue releases, joint debridements, joint fusions, joint manipulation via osteotomies (altering native bone alignment), and joint arthroplasties (or replacements). Multiple variables are considered when arriving at the appropriate surgical treatment plan, including patient age, which specific joint is involved, native joint anatomy, extent of osteoarthritis, functional expectations, and longevity of surgical intervention or implant.

To provide a context, osteoarthritis of the knee will be considered. Surgical options ranging from least to most invasive include joint arthroscopy with debridement and lavage, joint osteotomy to off-load isolated single compartmental disease, partial or compartmental joint replacement, and total joint replacement (Figure 2). Each procedure has an inherent duration of relief, postoperative rehabilitation, rate of disease progression, and life of the implant. These are the variables the surgeon and patient must discuss and understand prior to embarking on an operation.

\section{What Muslims need to know prior to joint replace- ment surgery}

As the largest senior population in America's history ages, the demand for joint replacement surgery will continue to grow. In 2006, more than 600,000 total knee and hip replacement surgeries were performed, which is up significantly from approximately 250,000 in the mid-1990s ${ }^{11}$ (Figure 2). During this period our first generation Muslim population is also aging and suffering from the effects of osteoarthritis. Therefore, through the standard algorithm for the management of osteoarthritis discussed previously, the discussion of whether to proceed with joint replacement surgery will be inevitable.

Joint replacement surgery can be performed for several joints in the body to treat advanced arthritis resistant to nonoperative modalities. It is most commonly performed for the hip and knee and has proven to be quite successful in providing excellent pain relief and function. The evolution of implant design and refinement of operative technique have gradually contributed to the success of these surgeries. But, they are not without surgical risks, such as infection, nerve injury, fracture, residual leg-length discrepancy, and continued pain. In addition, there are some changes in joint range of motion that can cause significant limitations to a patient's ability to perform salat (the Muslim ritual prayer) postoperatively.

In order to perform salat, an individual must be able to flex his hips at least 90 degrees and flex his knees approximately 150 degrees. After hip and knee replacement the normal range of motion of the patient's lower extremity will be either altered or limited or both, thereby limiting the patient's ability to perform salat in the standard format.

After hip replacement, the surgeon may limit the patient from bending the new prosthetic hip to no more than 90 degrees of flexion in order to avoid dislocation of the joint. This may limit a Muslim patient from performing ruku and sajdah in salat. Additional activity modifications must be made such as avoiding sitting in low seats and not allowing squatting on a low- or floor-level commode. The consequence of a prosthetic hip dislocation is significant, often requiring joint reduction in the operating room and increasing the risk for future instability.

After knee replacement, knee flexion of the new prosthetic knee is typically limited to no more than 130 degrees due to knee biomechanics, soft tissue restraints, and mechanical limitations of the implant. More commonly, patients typically recover 110-120 degrees of knee flexion postoperatively. This will limit a Muslim patient from performing sajdah and sitting during salat. Some implant companies are now producing "high-flex" total knee prosthesis that can offer up to 160 degrees of knee flexion. ${ }^{12}$ This has resulted in an overall greater improvement in postoperative knee flexion. Wohlrab et al identified an average postoperative knee range of motion of 122 degrees in their "high-flex" population compared to 107 degrees in their control population after 5 years. ${ }^{13}$ In addition, patients with greater preoperative knee range of motion have been able to maintain greater postoperative motion with the use of high-flex total knee prostheses. Hoang et al observed a postoperative range of motion of 138 degrees in 
Taiwan, ${ }^{14}$ while Tarabichi et al were able to obtain an average postoperative range of motion of 142 degrees in Dubai. ${ }^{15}$ Overall, Tarabichi et al were able to obtain full knee flexion by 2 years in $78 \%$ of the cases. ${ }^{15}$

Before embarking on hip or knee replacement, Muslim patients must be made aware of these range of motion limitations. Patients must be encouraged to discuss with their surgeons their expectant range of motion postoperatively in light of the techniques and implants to be employed and request their insight on whether they will be able to pray salat in the manner they wish. In the end, the decision to pursue joint replacement surgery will be improved if the patients' expectations are accurate and tempered.

\section{References}

1. Lawrence RC, Helmick CG, Arnett FC, et al. Estimates of the prevalence of arthritis and selected musculoskeletal disorders in the United States. Arthritis Rheum. 1998;41:778-99.

2. Shojania K, Esdaile JM, Greidanus N. Chapter 21 Arthritis. Orthopaedic Knowledge Update 8. American Academy of Orthopaedic Surgeons. 2005:229-44.

3. Felson DT, Anderson JJ, Naimark A, et al. Obesity and knee osteoarthritis. The Framingham Study. Ann Intern Med. 1988 Jul 1;109(1):18-24.

4. Coleman EA, Buchner DM, Cress ME, et al. The relationship of joint symptoms with exercise performance in older adults. J Am Geriatr Soc. 1996;44(1):14-21.

5. Richy F, Bruyere O, Ethgen O, et al. Time-dependent risk of gastrointestinal complications induced by non-steroidal anti-inflammatory drug use: a consensus statement using a meta-analytic approach Ann Rheum Dis. 2004;63(7):759-66.

6. Silverstein FE, Faich G, Goldstein JL, et al. Gastrointestinal toxicity with celecoxib vs nonsteroidal anti-inflammatory drugs for osteoarthritis and rheumatoid arthritis: the CLASS study: A ran- domized controlled trial. Celecoxib Long-term Arthritis Safety Study. JAMA. 2000 Sep 13;284(10):1247-55.

7. Bombardier C, Laine L, Reicin A, et al. Comparison of upper gastrointestinal toxicity of rofecoxib and naproxen in patients with rheumatoid arthritis. VIGOR Study Group. N Engl J Med. 2000 Nov 23;343(21):1520-8

8 Hochberg MC, Altman RD, Brandt KD, et al. Guidelines for the medical management of osteoarthritis. Part II. Osteoarthritis of the knee. American College of Rheumatology. Arthritis Rheum. 1995;38 (11):1541-6.

9. Ravaud P, Moulinier L, Giraudeau B, et al. Effects of joint lavage and steroid injection in patients with osteoarthritis of the knee: results of a multicenter, randomized, controlled trial. Arthritis Rheum. 1999;42(3):475-82.

10. Leopold SS, Redd BB, Warme WJ, et al. Corticosteroid compared with hyaluronic acid injections for the treatment of osteoarthritis of the knee: A prospective, randomized trial. J Bone Joint Surg Am. 2003;85-A(7):1197-203.

11. Joint replacement: 1,001 patients tell you what your doctor can't.. Consumer Reports, 2006;71:15-17. 12. Laskin RS. The effect of a high-flex implant on postoperative flexion after primary total knee arthroplasty. Orthopedics. 2007 Aug;30(8 Suppl):868.

13. Wohlrab D, Hube R, Zeh A, et al. Clinical and radiological results of high flex total knee arthroplasty: a 5 year follow-up. Arch Orthop Trauma Surg. Epub 2008 Jun 3.

14. Huang HT, Su JY, Wang GJ. The early results of high-flex total knee arthroplasty: a minimum of 2 years of follow-up. J Arthroplasty. 2005 Aug;20(5):674-9.

15. Tarabichi S, Tarabichi Y, Tarabishy AR, et al. Importance of full flexion after total knee replacement in Muslim's daily lifestyle. J Islam Med Assoc. 2006;38(1):17-22. 